Highly Energetic Physical Processes and

Mechanisms for Emission from Astrophysical Plasmas

IAU Symposium, Vol. 195, 2000

P. C. H. Martens, S. Tsuruta, and M. A. Weber, eds.

\title{
Statistical Properties of Magnetic Separators in Model Active Regions
}

\author{
B. T. Welsch and D. W. Longcope \\ Department of Physics, Montana State University - Bozeman, \\ Bozeman, MT 59717 - 3840, U.S.A.
}

\begin{abstract}
Transient brightenings" (or "microflares") regularly deposit $10^{27}$ ergs of energy in the solar corona, and account for perhaps $20 \%$ of the active corona's power (Shimizu 1995). We assume these events correspond to episodes of magnetic reconnection along magnetic separators in the solar corona. Using the techniques of magnetic charge topology, we model active region fields as arising from normally distributed collections of "magnetic charges", point-like sources/sinks of flux (or field lines). Here, we present statistically determined separator (X-ray loop) lengths, derived from first principles. We are in the process of statistical calculations of heating rates due to reconnection events along many separators.
\end{abstract}

A positive (negative) charge's separatrix surface divides field lines which begin (end) at that charge from those beginning (ending) at other charges. Accordingly, separatrix field lines begin (end) at a "positive" ("negative") magnetic null $(\mathbf{B}(\mathbf{x})=0)$, since they enclose a positive (negative) charge's flux. The intersection of a negative charge's separatrix surface with the photosphere is shown in Figure 1.

A magnetic separator is the field line formed along the intersection of a positive and negative charge's separatrices, and so must run from a positive null to a negative one. Previous theoretical work (Longcope 1996, and references therein) suggests that reconnection should occur along these separators, which are the three-dimensional generalizations of X-type points in two dimensions.

We consider the interaction of a single "test" charge with a "background" active region field which arises from vertical flux which, on average, is normally distributed (grayscale in Figure 1):

$$
\left\langle B_{z}(x, y)\right\rangle=\frac{q_{0} N_{+}}{\delta^{2}} e^{-\left(\left(x^{2}+y^{2}\right) / 2 \delta^{2}\right)}-\frac{q_{0} N_{-}}{\delta^{2}} e^{\left.-\left(\left(x-x_{0}\right)^{2}+y^{2}\right) / 2 \delta^{2}\right)},
$$

where $N_{ \pm}$parametrizes the background field strength, and $\delta$ is the background field's Gaussian width ( $N=100$ and $\delta=40 \mathrm{Mm}$ are typical solar values).

We begin with $N_{-}=0$ for simplicity. We model the interaction with two techniques: we generate distributions (a Monte Carlo approach) of charges (+ and $\times$ in Figure 1, with $N_{+}=N_{-}$), and we use mean-field averaging (grayscale in Figure 1).

Separator lengths should depend upon both the test charge's distance $r_{\mathrm{t}}$ from the center of the field distribution, and upon that distribution's relative strength, parametrized by $N$. Both approaches give similar expectation values 


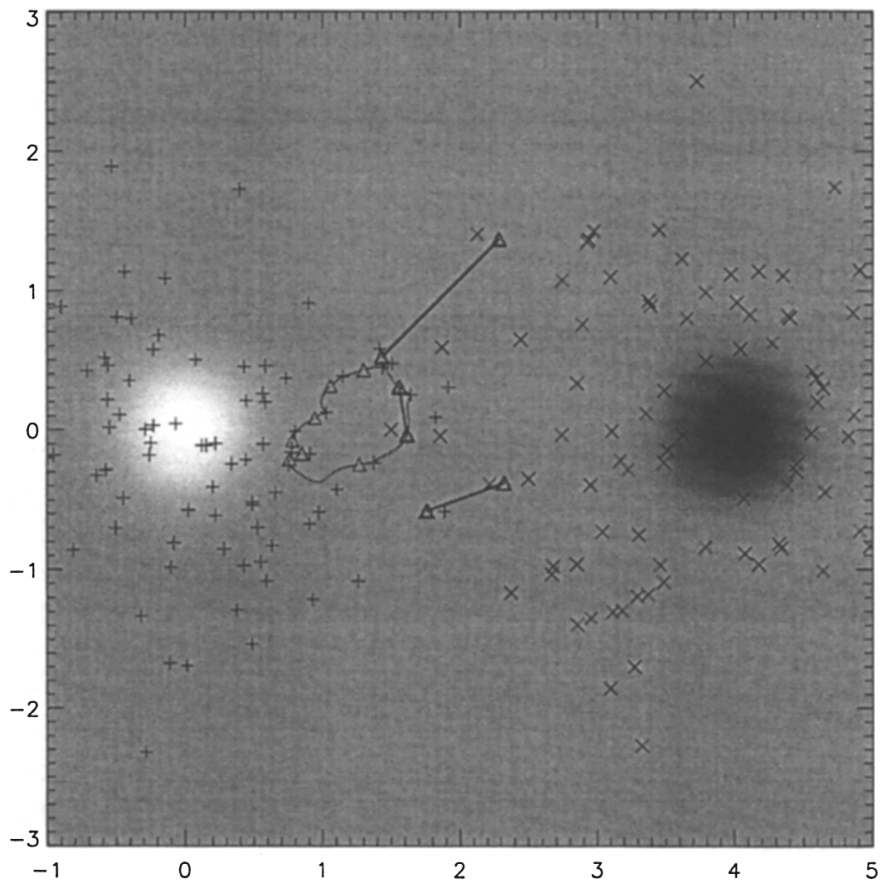

Figure 1. A model solar active region, composed of $N$ discrete positive $(+)$ and $N$ negative $(x)$ charges. Grayscale illustrates normal probability distributions used to generate random charge distributions. The intersection of one negative charge's separatrix with the photosphere is shown (thin solid). Selected magnetic nulls are plotted $(\Delta)$, as well. Finally, the inferred projection of selected separator loops onto the photosphere is also plotted (thick solid).

for separator lengths,

$$
<\ell>\simeq 2.75 \delta \exp \left(\alpha r_{\mathrm{t}}\right) / \sqrt{N},
$$

with $\alpha=.35(.65)$ in the mean-field (Monte Carlo) case. This prediction of loop lengths, derived from first principles, can be compared with observations.

We are now extending this study to the $N_{+}=N_{-}$case (Figure 1). Portions of this work are described in more detail in Welsch \& Longcope (1999).

\section{References}

Longcope, D. W. 1996, Solar Phys., 169, 91

Shimizu, T. 1995, PASJ, 47, 251

Welsch, B. T., \& Longcope, D. W. 1999, ApJ, 522, 1117 